\title{
Leitlinien für Forschungsberichte: Deutschsprachige Übersetzungen von CONSORT 2010, PRISMA und STARD
}

Vollständig, klar und transparent muss der Bericht über die Ergebnisse einer Studie sein, damit diese Studie vom Leser genau beurteilt werden kann [6]. Die Elemente, die für die Beurteilung der Qualität und der Resultate zentral sind, werden in Leitlinien für Forschungsberichte zusammengefasst. Die Deutsche Medizinische Wochenschrift hat jetzt die Übersetzung der drei wichtigsten dieser Leilinien frei zugänglich online publiziert, nämlich

- das CONSORT 2010 Statement [13]: Consolidated Statement f Reporting Trials (konsolidierte Leitlinie zum Berichten randomisierter Studien im Parallelgruppendesign), das PRISMA Statement [8]: Preferred Reporting Items for Systematic reviews and MetaAnalyses (Bevorzugte Report Items für systematische Übersichten und Meta-Analysen) und

das STARD Statement [3]: Standards for Reporting of Diagnostic Accuracy (Standards zum Berichten der diagnostischen Genauigkeit).

Das CONSORT Statement kann als „Ur-Leitlinie“ für Forschungsberichte angesehen werden. Entwickelt Anfang der 1990er Jahre wurde fünf Jahre später eine erste Weiterentwicklung publiziert, die auch in deutscher Sprache vorliegt $[1,10]$. Vor kurzem wurde eine weitere Aktualisierung parallel in mehreren englischsprachigen Fachzeitschriften veröffentlicht [12]. CONSORT 2010 besteht aus einer 25-Punkte-Checkliste sowie einem Flussdiagramm und ist eine Leitlinie für Berichte randomisierter Studien jeglicher Art. Allerdings wird darin auf das häufigste Studiendesign, individuell randomisierte Parallelgruppenvergleiche mit zwei Parallelgruppen fokussiert. Andere Studientypen wie Cluster-randomisierte Studien erfordern ein unterschiedliches Maß zusätzlicher Informationen. Diese sind in entsprechenden Erweiterungen verfügbar, welche von www.consort-statement.org heruntergeladen werden können. Diese Erweiterungen stehen allerdings nicht in deutscher Sprache zur Verfügung. Als Online-Material steht zur Illustration die Anwendung der neuen 25-PunkteCheckliste des CONSORT 2010 Statements sowie der Erweiterung des CONSORT Statements auf Zusammenfassungen in Zeitschriftenartikeln und Tagungsbänden auf die Publikation von Richter et al. zur Verfügung $[4,5,11]$.

Für systematische Reviews und Meta-Analysen liegt mit dem PRISMA Statement [9] die Weiterentwicklung des QUORUM Statements („QUality Of Reporting Of Meta-analyses, Qualität des Re- ports von Meta-Analysen“) [7] vor, in dem eine Reihe konzeptueller und praktischer Fortschritte bei systematischen Übersichten adressiert werden. Der Schwerpunkt wurde bei PRISMA auf randomisierte Studien gelegt. Doch kann das PRISMA-Statement auch als Grundlage für das Berichten von systematischen Übersichten anderer Studien, insbesondere von Interventionsstudien verwendet werden.

Schließlich steht mit dem STARD Statement eine Leitlinie zur Verfügung, die eine Hilfestellung beim Berichten und Beurteilen von Diagnosestudien in Form einer 24-Punkte-Checkliste und eines Flussdiagramms gibt [2].

Die Leitlinien erfüllen zwei Aufgaben: Zum einen helfen sie Autoren von Fachartikeln, vollständige und transparente Berichte zu erstellen. Zum anderen können Leser Leitlinien-Elemente zur Einschätzung der Qualität einer publizierten Studie verwenden. Leser können sich bei der kritischen Evaluation von Fachartikeln an diesen Leitlinien orientieren, um die Qualität und Übertragbarkeit einer Studie einzuschätzen. Allerdings muss bei der kritischen Beurteilung eines Artikels stets der Unterschied zwischen der Qualität des Berichtens und der Qualität der eigentlichen Studie beachtet werden [6].

Danksagung: Diese Arbeit entstand während des Forschungsfreisemesters von $\mathrm{AZ}$ an dem Institut Montefiore der Universität Lüttich (Belgien). AZ dankt den Kollegen am Institut Montefiore sehr herzlich für die Möglichkeit, dort zu arbeiten. Die Arbeit entstand während der Vorbereitung der Autoren auf den Kursteil Evidenzbasierte Medizin im Rahmen des Kurses Ärztliches Qualitätsmanagement an der Akademie für Fortbildung und Weiterbildung der Landesärztekammer Hessen (Sigrid Blehle, Dr. Roland Kaiser).

Autorenerklärung: Die Autoren erhalten ein Honorar von der Akademie für Fortbildung und Weiterbildung der Landesärztekammer Hessen für das Erstellen von Unterlagen für den Kursteil Evidenzbasierte Medizin im Rahmen des Kurses Ärztliches Qualitätsmanagement.

\section{Literatur}

1 Begg C, Cho M, Eastwood S et al. Improving the quality of reporting of randomized controlled trials. The CONSORT statement. JAMA 1996; 276: 637-639

2 Bossuyt PM, Reitsma JB, Bruns DE et al. Towards complete and accurate reporting of studies of diagnostic accuracy: the STARD initiative. Fam Pract 2004; 21 : 4-10

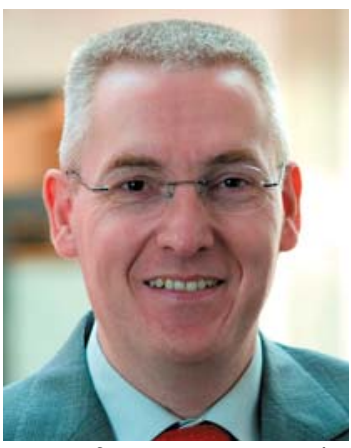

Prof. Dr. rer. nat. A. Ziegler

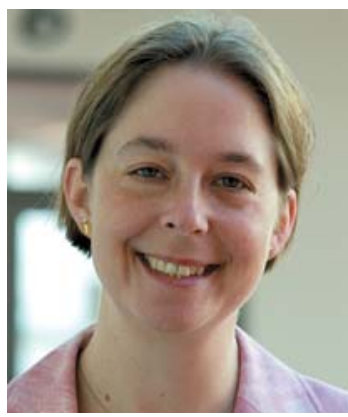

Prof. Dr. rer. biol. hum. I. R. König

\section{A. Ziegler}

I. R. König

Medizinisches Publizieren

Institut

Institut für Medizinische Biometrie und Statistik, Universität zu Lübeck

Bibliografie

Dol $10.1055 / \mathrm{s}-0031-1272535$

Dtsch Med Wochenschr 2011; 136: 357-358 - (c) Georg Thieme Verlag KG Stuttgart New York · ISSN 0012-0472

Korrespondenz

Univ.-Prof. Dr. rer. nat. Andreas Ziegler

Institut für Medizinische Biometrie und Statistik Universität zu Lübeck Universitätsklinikum SchleswigHolstein, Campus Lübeck Maria-Goeppert-Str. 1 23538 Lübeck

Tel. 0451-500/2780

Fax 0451-500/2999

eMail

ziegler@imbs.uni-luebeck.de 
3 Ziegler A, König IR. Vollständiges und präzises Berichten von Studien zur diagnostischen Genauigkeit: Die STARD Initiative. Dtsch Med Wochenschr 2011; 136: e16

4 Hopewell S, Clarke M, Moher D et al. CONSORT for reporting randomised trials in journal and conference abstracts. Lancet 2008; 371: 281-283

5 Hopewell S, Clarke M, Moher D et al. CONSORT for reporting randomized controlled trials in journal and conference abstracts: explanation and elaboration. PLoS Med 2008; 5: e20

6 Meerpohl JJ, Blümle A, Antes G, Elm E. Leitlinien für Forschungsberichte sind auch für Leser medizinischer Fachartikel hilfreich: CONSORT, STARD, STROBE \& Co. Dtsch Med Wochenschr 2009; 134: 2078-2083
7 Moher D, Cook DJ, Eastwood S et al. Improving the quality of reports of meta-analyses of randomised controlled trials: the QUOROM statement. Quality of Reporting of Meta-analyses. Lancet 1999; 354: 1896-1900

8 Ziegler A, Antes G, König IR. Bevorzugte Report Items für systematische Übersichten und Meta-Analysen: Das PRISMA Statement. Dtsch Med Wochenschr 2011; 136: e9

9 Moher D, Liberati A, Tetzlaff J, Altman DG. Preferred reporting items for systematic reviews and meta-analyses: the PRISMA statement. BMJ 2009; 339: b2535

10 Moher D, Schulz KF, Altman DG für die CONSORT Gruppe. Das CONSORT Statement Überarbeitete Empfehlungen zur Qualitätsverbesserung von Reports randomisierter Studien im Parallel-Design. Dtsch Med Wochenschr 2004; 129: T16-T20
11 Richter $H$, Kraft K, Kleinwechter $H$ et al. Telefonintervention bei Typ-2-Diabetikern: $\mathrm{Zu}$ nahme der körperlichen Aktivität und Reduktion von kardiovaskulären Risikofaktoren. Dtsch Med Wochenschr 2008; 133: 2203-2208

12 Schulz KF, Altman DG, Moher D. CONSORT 2010 statement: updated guidelines for reporting parallel group randomized trials. Ann Intern Med 2010; 152: 726-732

13 Pittler M, Blümle A, Meerpohl IJ, Antes G. CONSORT 2010: Aktualisierte Leitlinie für Berichte randomisierter Studien im Parallelgruppen-Design. Dtsch Med Wochenschr 2011; 136: e20

\section{Im DMW Supplement Statistik 2007 online erschienene Beiträge zum Thema}

Diese Beiträge finden Sie unter www.thieme-connect.de/ejournals/toc/dmw/27281

Lange, S.; Bender, R.:

\section{Median oder Mittelwert?}

Lange, S.; Bender, R.:

Quantile, empirische Verteilungsfunktion und Box Plot

Lange, S.; Bender, R.:

Variabilitätsmaße

Lange, S.; Bender, R.:

\section{Das Histogramm}

Lange, S.; Bender, R.:

Lineare Regression und Korrelation

Bender, R.; Lange, S.:

Die Vierfeldertafel

Bender, R.; Lange, S.:

Was ist der p-Wert?

Bender, R.; Lange, S.:

Was ist ein Konfidenzintervall?

Lange, S.; Bender, R.:

Was ist ein Signifikanztest? Allgemeine Aspekte

Bender, R.; Lange, S.:

Verlaufskurven

Bender, R.; Lange, S.; Ziegler, A.:

Wichtige Signifikanztests

Bender, R.; Lange, S.; Ziegler, A.:

Multiples Testen
Bender, R.; Ziegler, A.; Lange, S.:

Multiple Regression

Bender, R.; Ziegler, A.; Lange, S.:

Logistische Regression

Ziegler, A.; Lange, S.; Bender, R.:

Überlebenszeitanalyse: Eigenschaften und

Kaplan-Meier Methode

Ziegler, A.; Lange, S.; Bender, R.:

Überlebenszeitanalyse: Der Log-Rang-Test

Ziegler, A.; Lange, S.; Bender, $R$.

Überlebenszeitanalyse: Die Cox-Regression

Klug, S. J.; Bender, R.; Blettner, M.; Lange, S.:

Wichtige epidemiologische Studientypen

Ziegler, A.; Lange, S.; Bender, R.:

Systematische Übersichten und Meta-Analysen

Lange, S.; Bender, R.; Ziegler, A.:

Äquivalenzstudien und Nicht-Unterlegenheitsstudien

Bender, R.; Ziegler, A.; Lange, S.:

Varianzanalyse

Bender, R.; Grouven, U.; Ziegler, A.:

Varianzanalyse für Messwertwiederholungen

Grouven, U.; Bender, R.; Ziegler, A.; Lange, S.:

Der Kappa-Koeffizient

Grouven, U.; Bender, R.; Ziegler, A.; Lange, S.: Vergleich von Messmethoden 